\title{
Novel Methods for Recording Stress-Strain Curves in Proton Irradiated Material
}

\begin{abstract}
Albert D. Smith ${ }^{1 *}$, Jack M. Donoghue ${ }^{1}$, Alistair J. W. Garner ${ }^{1}$, David Lunt ${ }^{1}$, Allan Harte ${ }^{1,2}$, Keith Wilford ${ }^{3}$, Philip J. Withers ${ }^{1,4}$ \& Michael Preuss ${ }^{1,4}$

Proton irradiation is often used as a proxy for neutron irradiation but the irradiated layer is typically $<50 \mu \mathrm{m}$ deep; this presents a problem when trying to obtain mechanical test data as a function of irradiation level. Two novel methodologies have been developed to record stress-strain curves for thin proton-irradiated surface layers of SA-508-4N ferritic steel. In the first case, in-situ loading experiments are carried out using a combination of $\mathrm{X}$-ray diffraction and digital image correlation on the near surface region in order to measure stress and strain, thereby eliminating the influence of the non-irradiated volume. The second approach is to manufacture small-scale tensile specimens containing only the proton irradiated volume but approaching the smallest representative volume of the material. This is achieved by high-speed focused ion beam (FIB) milling though the application of a Xe+ $\mathrm{Plasma}^{+}$-FIB (PFIB). It is demonstrated that both techniques are capable of recording the early stage of uniaxial flow behaviour of the irradiated material with sufficient accuracy providing a measure of irradiation-induced shift of yield strength, strain hardening and tensile strength.
\end{abstract}

In order to increase the power capacity and operational life of modern reactor designs, a better understanding of the performance of materials under high levels of irradiation damage is required. Displacement damage leads to degradation of mechanical properties through irradiation-induced hardening and reduction in strain hardening and strain to failure ${ }^{1,2}$. The most reliable method of testing materials behaviour under reactor conditions is achieved by placing surveillance specimens within an operating or test reactor, which are removed and tested periodically ${ }^{3}$. However, the high cost and long duration of these tests can be prohibitive ${ }^{4}$. The low displacement efficiency of neutrons leads to experiments running from months to years in order to reach relevant levels of damage. In addition, high post-irradiation activity can require long cooling-off periods, or expensive radiation handling protocols, to safely perform off-site examination, increasing test duration further ${ }^{5}$. This leads to a long turnaround time for specimens and can be prohibitive to studies requiring a wide range of irradiating conditions or new alloy designs. As a result, more cost effective, alternative forms of irradiation, particularly proton and heavy ion irradiation, have been developed in order to mimic the effects of neutron irradiation over shorter timescales with reduced activation.

Protons produced by a spallation source typically have energies in the order of $100 \mathrm{~s}$ of $\mathrm{MeV}$, inducing displacement damage similar to the effects of neutrons. Such high kinetic energy has the advantage of through-thickness irradiation of bulk specimens, so can be analysed using standard mechanical testing methods ${ }^{6-12}$. However, this approach suffers from the same limitations as neutron irradiation, in terms of poor displacement efficiency and high residual post irradiation activity. This drives up experimental expense and limits the availability of equipment. Lower energy proton beams (e.g. generated by Pelletron) induce similar displacement damage effects with a reduced post-irradiation activity, allowing for easy handling, transportation and testing ${ }^{13,14}$. The reduced energy of the incident beam is more efficient at generating displacement damage and so displacement damage rates can be increased by up to an order of magnitude. Depending on the energy, low energy protons can attain damage levels in a matter of hours that would take months to achieve in a test reactor or on a spallation beamline. However, the benefits of low energy proton irradiation comes at the expense of significantly reduced penetration depth ${ }^{15}$.

${ }^{1}$ School of Materials, The University of Manchester, Manchester, M13 9PL, UK. ${ }^{2}$ United Kingdom Atomic Energy Authority Culham Science Centre, Abingdon, OX14 3DB, UK. ${ }^{3}$ Rolls-Royce plc, Raynesway, Derby, DE21 7WA, UK. ${ }^{4}$ Henry Royce Institute, School of Materials, The University of Manchester, Manchester, M13 9PL, UK. *email: albert. smith@manchester.ac.uk 


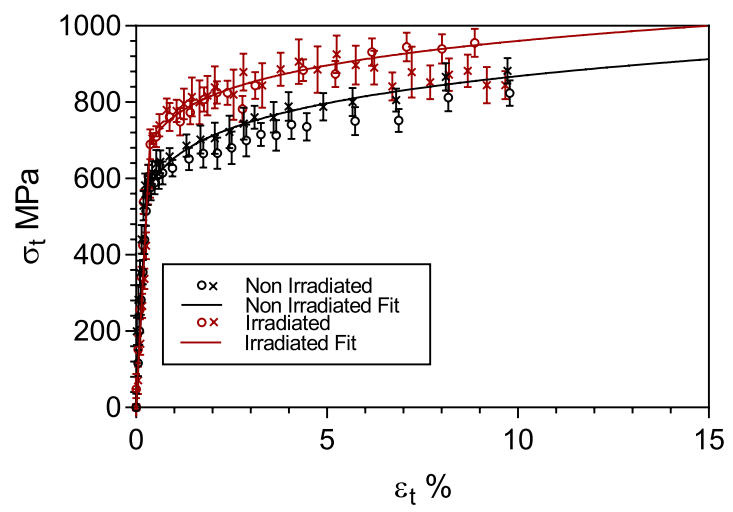

Figure 1. True stress - true strain curves generated by XRD/ DIC technique for irradiated and non-irradiated specimens, error bars are gradient error in $\sin ^{2} \Psi$ vs. d-spacing plot. Red markers are irradiated, black markers are non-irradiated collected in ref. ${ }^{35}$, with crosses and open circles representing different specimens. Solid lines depict fitted curves assuming power-law hardening from each technique for irradiated and non-irradiated states.

The limited penetration depth of proton irradiation makes measurement of mechanical properties difficult using established techniques ${ }^{16,17}$. Over the last decade, the use of $\mathrm{Ga}^{+}$focussed ion beam (FIB) instruments has allowed for the preparation of small scale samples from proton irradiated layers ${ }^{18-23}$. The mechanical properties of these samples are then tested using a MEMS chip or piezo-actuated test rig without contributions from the non-irradiated volume. This development has allowed studies to take advantage of the increased dose rates and to probe changes in properties previously only attainable using indentation testing ${ }^{24-28}$. However, milling rates are slow because for $\mathrm{Ga}^{+}$FIBs the useable milling currents are limited due to the point source of $\mathrm{Ga}^{+}$ions. Consequently, specimen diameters achievable in a practical time frame are limited to $\sim 10 \mu \mathrm{m}$. For most engineering materials, the maximum achievable scale is therefore in the order of single to only a few grains. Hence, the technique is most applicable to single and bi-crystal investigations rather than representing the bulk response of polycrystalline specimens. It has been demonstrated that small scale specimens exhibit an increased hardening inversely proportional to specimen diameter ${ }^{29-33}$. In order to obtain a bulk response, specimens require a minimum length scale sufficient to overcome size reduction effects. The optimum specimen size would have the smallest representative volume (SRV), which would retain the benefits of scale reduction whilst exhibiting bulk behaviour $^{34}$.

Recent work by the authors has explored two techniques to increase the sampling volume in low energy proton irradiated samples prepared for mechanical testing ${ }^{35,36}$. The first, an adaptation of the technique outlined by Foecke et al. ${ }^{37}$, combines in-situ X-ray diffraction stress measurement and digital image correlation (DIC) to construct uniaxial flow curves ${ }^{35}$. Laboratory-based $\sin ^{2} \Psi$ diffraction stress measurement relies on the limited $\mathrm{X}$-ray penetration depth, which for steels is of the same order as the proton irradiated layer $(\sim 35 \mu \mathrm{m} \text { at } 3 \mathrm{MeV})^{35}$. Calibration of the lattice response to applied stress, using the methodology outlined in ASTM E1426 $-98^{38}$, allows for the calculation of stresses in the same material under the assumption that the lattice response to applied stress is consistent between tests. Strain is calculated using DIC on optical images collected in the gauge area in parallel to the stress measurements. DIC allows for non-contact measurement of the in-plane (total) strain on the surface and therefore both stress and strain data are collected from the near surface region that is affected by irradiation hardening. The second method exploits the significantly faster milling rates that can be achieved for plasma $\mathrm{Xe}^{+}$ focussed ion beam (PFIB) technology compared to $\mathrm{Ga}^{+}$FIBs due to the higher milling currents ${ }^{39}$. This enables large scale machining of material at milling rates some 100 times that of $\mathrm{Ga}^{+} \mathrm{FIB}^{40,41}$. A methodology has been developed for the manufacture of samples with a gauge cross sections in the $90 \mu \mathrm{m}^{2}$ range $^{36}$. It has been demonstrated that such specimens exhibit a proof stress comparable to a standardised bulk test, however, they did display a size-dependent plastic deformation response ${ }^{36}$. A methodology has been developed for the manufacture of samples with dimensions approaching the SRV, with a gauge section in the meso-scale exhibiting bulk behaviour in all aspects except strain hardening ${ }^{36}$. The present study aims to apply and compare side by side these novel techniques for the first time in order to record the mechanical response of $3 \mathrm{MeV}$ proton irradiated SA508-4N steel (a candidate RPV steel), irradiated to a depth of approximately $30 \mu \mathrm{m}$.

\section{Results}

A comparison between the flow curves generated from the XRD/DIC technique in the irradiated region, irradiated using $3 \mathrm{MeV}$ protons to the range of around $10 \mathrm{mdpa}$ according to SRIM calculations at $330 \pm 3^{\circ} \mathrm{C}$ and a flux of $2.3 \times 10^{14} \mathrm{~cm}^{-2} \mathrm{~s}^{-1}$, and from the non-irradiated material from ref. ${ }^{36}$ is shown in Fig. 1 . The proof stresses of the irradiated samples were obtained using the standard $0.2 \%$ offset, providing an average value of $770 \pm 29 \mathrm{MPa}$, whereas the proof stress of the non-irradiated material was $633 \pm 22 \mathrm{MPa}$. This value agrees well with the estimated increase in yield stress of $110 \mathrm{MPa}$ inferred from indentation testing, which was calculated using the relationship from ref. ${ }^{25}$ : 


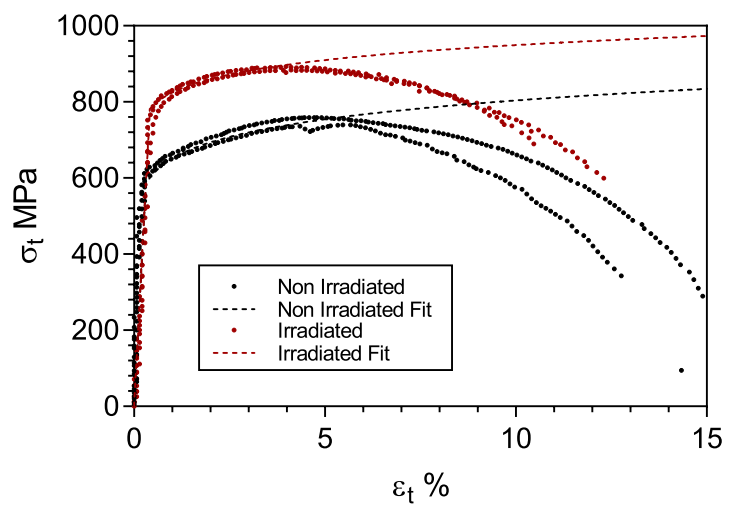

Figure 2. Stress - strain plots of $i n$-situ testing of small-scale specimens prepared by PFIB. Red markers are irradiated and black markers are non-irradiated collected in ref. ${ }^{36}$. Dashed lines depict fitted curves assuming power-law hardening from each technique for irradiated and non-irradiated states. True stress and true strain are only valid during uniform plastic deformation, so can be considered as engineering stress and strain beyond the plastic instability strain.
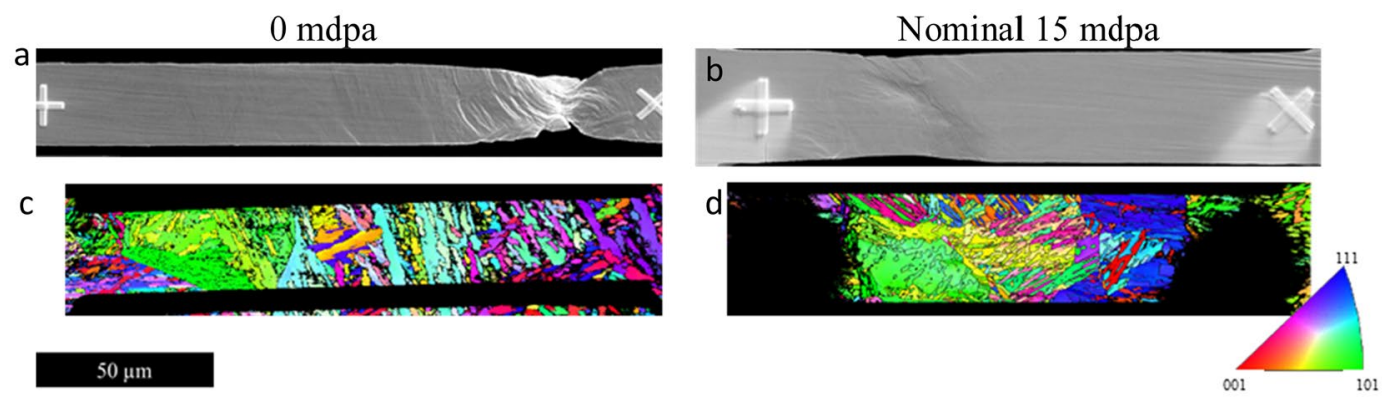

Figure 3. Post necking behaviour of non-irradiated and irradiated micro-tensile specimens. (a,b) Secondary electron images taken prior to failure; (c,d) Electron backscattered diffraction orientation maps collected prior to testing of non-irradiated and irradiated specimens (displayed in the inverse pole figure colour scheme) projects plane normal parallel to the loading direction; a \& c are adapted from ${ }^{36}$.

$$
\Delta \sigma_{y}=3.03 \Delta H_{V}
$$

where, $\Delta \sigma_{y}$ is the calculated increase in yield stress; $\Delta H_{V}$ is the measured increase in Vickers hardness due to irradiation damage and 3.03 is the correlation function for ferritic steel ${ }^{25}$.

Strain hardening was calculated for the irradiated specimens using the log-log gradient of the plastic regime between proof and peak stress. The stress-strain curves exhibited a slight reduction of $\sim 0.013$ in strain hardening, relative to the non-irradiated material ( $\sim 11 \%$ change). Tensile strength, taken as the peak stress, remained unchanged at $\sim 875 \mathrm{MPa}$, while the strength coefficient (K) was observed to decrease by $89 \mathrm{MPa}$ from $1120 \mathrm{MPa}$. Fitted curves generated using Hooke's law and a power law hardening model are shown in Fig. 1, with plastic behaviour characterised by the Hollomon equation:

$$
\sigma_{t}=K \varepsilon^{n}
$$

where $\sigma_{t}$ is true stress, $\mathrm{K}$ is the strength coefficient and $n$ is the strain hardening exponent.

Figure 2 illustrates the flow curves recorded from the micro-tensile specimens prepared from the proton irradiated region using the $\mathrm{Xe}^{+}$PFIB method described above. Here the proof stress was recorded as $807 \pm 9 \mathrm{MPa}$, exhibiting an irradiation hardening of $161 \pm 9 \mathrm{MPa}$. This is approximately $24 \mathrm{MPa}$ higher than the average value of hardening reported using the XRD and DIC technique, and $\sim 50 \mathrm{MPa}$ more than that inferred from indentation testing. The strain hardening exponent was calculated to be 0.06 , a reduction of 0.034 relative to the non-irradiated state. It must be emphasised that the strain hardening exponent recorded in the non-irradiated specimen is already lower for the micro-tensile specimen than in the bulk samples ${ }^{36}$. The exponent is approximately $21 \%$ lower than that recorded using the XRD-DIC method and the standardised method ${ }^{35}$. Tensile strength was measured to be to $866 \pm 3 \mathrm{MPa}$ using this technique, corresponding to an increase relative to the non-irradiated state of $135 \mathrm{MPa}$; furthermore, the strength coefficient was increased to $1093 \mathrm{MPa}$ from $992 \mathrm{MPa}$.

SEM images (Fig. 3a,b) of each PFIB specimen prior to failure show some apparent differences between the non-irradiated and irradiated specimen, Fig. 3c,d show corresponding orientation maps for each of the images 

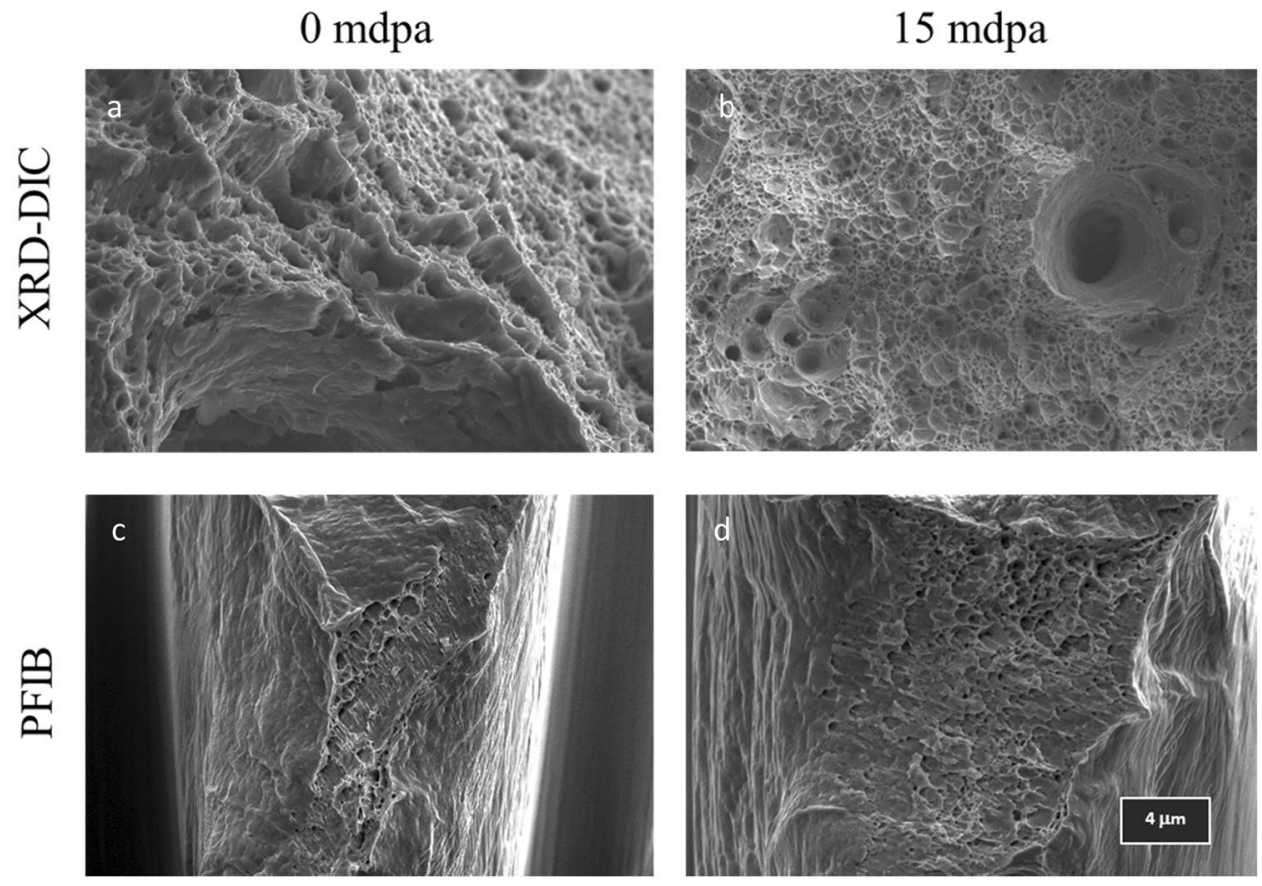

Figure 4. Secondary electron images of fracture surfaces for each sample (a) non-irradiated XRD/DIC; (b) irradiated XRD/DIC; (c) micro-tensile (PFIB) non-irradiated (d) micro-tensile (PFIB) irradiated.

shown. The irradiated specimen displays planar slip in the region of the neck which is indicative of dislocation channelling due to defect clearing during deformation, while the non-irradiated shows more diffuse homogeneous deformation ${ }^{42}$. Fracture surfaces for irradiated and non-irradiated specimens, tested using both techniques, are displayed in Fig. 4. The region displayed for the irradiated sample, tested using the combination of XRD and DIC, is the flat portion of the dose profile. All examples illustrate that both non-irradiated and irradiated samples failed in the same way, by ductile void coalescence. However, fractography of the micro-tensile samples highlights the triaxial stress state at the neck during failure. This is amplified due to the removal of constraint, resulting in drawn out elongated cavitation, which extends in the direction of shearing. It is also notable that the reduction in area is significantly larger in the non-irradiated sample than the irradiated, corresponding to $95 \%$ and $71 \%$ respectively.

\section{Discussion}

Both techniques have demonstrated that they are capable of recording a change in mechanical properties induced by displacement damage from proton irradiation. An increase in yield stress and decrease in strain hardening was documented using both XRD-DIC and micro-tensile testing.

Due to the variance in relative sampling volumes for each technique, the recorded values of yield shift are slightly different despite being irradiated under the same conditions. This difference corresponds to a variation of nearly $40 \mathrm{MPa}$, with the specimens prepared using PFIB having the largest yield shift. Although the samples prepared using PFIB accumulate some surface damage due to ion beam milling, previous observations have shown that the damage layer is approximately $40 \%$ shallower compared to that induced using $\mathrm{Ga}^{+}$FIB, being in the order of $10 \mathrm{~s}$ of nano meters ${ }^{43,44}$. This represents approximately $5 \times 10^{-3} \%$ of the sample volume which is not thought to contribute significantly to the measured shift. Preparation of the specimens will also introduce heat, temperature increases of $100^{\circ} \mathrm{C}$ are predicted at $20 \mathrm{nA} \mathrm{\mu m}^{-1}$ for $\mathrm{Ga}^{+} \mathrm{FIB}$ milling of materials with a similar thermal conductivity to the alloy in the present study, with the range of the heated zone calculated as approximately twice the diameter of the beam spot ${ }^{45}$. In addition, post irradiation annealing studies of ferritic steel alloys have shown that below $300{ }^{\circ} \mathrm{C}$ there is little defect annihilation from increased thermal mobility ${ }^{46}$. While it is likely that high current milling with $\mathrm{Xe}^{-}$ions lead to higher temperatures, the annealing footprint would be so localised that it is not expected to have an effect on the mechanical properties recorded. The relatively small differences between proof stresses in the non-irradiated dataset indicates that the variation evident in the irradiated sample is not a characteristic intrinsic to the method of preparation. This variation in yield stress is considered to be due to the difference in relative sampling volumes between the techniques, each effectively measuring a different dose. Figure 5 illustrates this; the area shaded in grey highlights the region sampled in the micro-tensile specimens and the plots shaded red through to black illustrate the attenuation of X-rays at each $\Psi$-tilt. The penetration depth as a function of tilt was calculated using ${ }^{47}$ :

$$
G_{x}=1-\exp \left\{-\mu x\left[\frac{1}{\sin (\theta+\psi)}+\frac{1}{\sin (\theta-\psi)}\right]\right\}
$$




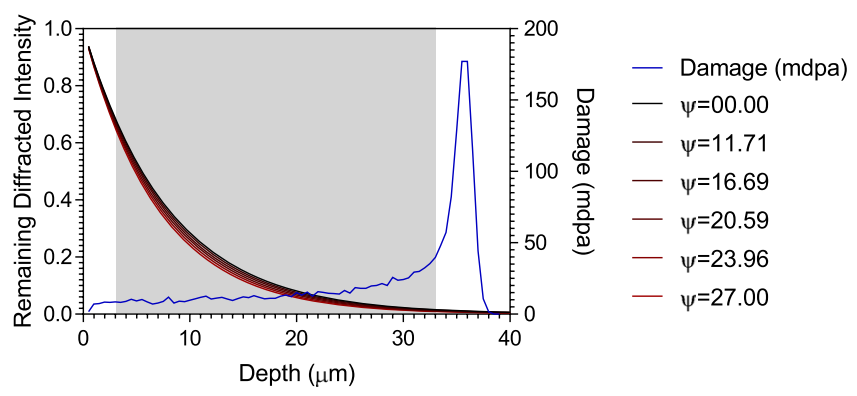

Figure 5. Technique dependent sampling depth relative to the SRIM damage profile for both techniques; shaded region corresponds to sampling range of PFIB micro-tensile specimens.

where $G_{x}$ is the total diffracted intensity at a depth of $x ; \mu$ is the linear absorption coefficient; $\theta$ is the diffraction half angle and $\psi$ is the tilt angle relative to the sample surface.

In contrast, the sampling volume of the micro-tensile specimens were sampled from the region ranging from $3-33 \mu \mathrm{m}$, with uniform sampling over this segment of the dose profile. Weighted dose $\left(D_{w}\right)$ was calculated by integration to take account of both the non-linear sampling of XRD and the non-linear dose profile simulated using SRIM:

$$
D_{w}=\frac{\sum D_{i} G_{i}}{\sum G_{i}}
$$

and

$$
D_{w}=\frac{\sum D_{i}}{N}
$$

where $D_{i}$ and $G_{i}$ are dose and diffracted intensity diffracted intensity respectively at each bin and $N$ is the number of bins. Bins were set at $500 \mathrm{~nm}$ increments, with $D$ and $G$ calculated as a trapezium to increase accuracy.

Each dose, weighted for $\Psi$-tilts, gave an average of 9.9 mdpa calculated for those samples measured using XRD. Dose was found to be inversely proportional to $\Psi$-tilt angle due to the larger penetration depth at lower angles and ranged by $0.5 \mathrm{mdpa}$ from $10.2 \mathrm{mdpa}$ at $\Psi=0^{\circ}$ to $9.7 \mathrm{mdpa}$ at $\Psi=27^{\circ}$. The range of doses were relatively narrow due to the exclusion of the stopping peak. Samples prepared using PFIB included some of the base of calculated damage peak, this gave a weighted dose of 15.6 mdpa. Given the weighted dose is $37 \%$ larger and the yield shift measured was greater in the specimens prepared by PFIB, the difference in proof stress is most likely due to the sampling volume.

Both techniques revealed a measured decrease in strain hardening due to irradiation damage. This behaviour is consistent with previous observations that show that irradiation damage has a deleterious effect as a function of $\operatorname{dos}^{48}$ up to the critical value where strain hardening drops to zero and necking occurs at yield ${ }^{49}$. There is a distinct difference between the measured values using both approaches, however this is not thought to be due to differences in the sampling volumes as with the yield shift.

The strain hardening exponent measured from stress strain curves recorded by XRD-DIC in the non-irradiated condition is comparable to that for the bulk ${ }^{35}$. However, when considering the differences in yield points and hardening rates of the non-irradiated "substrate" and irradiated layer, this may lead to a deviation from the results of a sample that was irradiated through thickness. The lattice response of dual-phase or composite materials during in-situ loading is analogous to the current study. Tensile deformation of these dual property materials will exhibit a linear response to applied stress up to the yield point of the softest constituent. Beyond which, load is understood to partition and will be transferred onto the harder of the constituents ${ }^{50-53}$. Hence, this will result in a larger stress recorded in the harder constituent of a dual property material than would be the case at the same level of strain if the same constituent were isolated. This has implications on the validity of the measured strain hardening in the irradiated layer. Due to partitioning, each increment of strain would result in a larger level of stress, which in turn will affect the calculated strain hardening parameter and is further complicated when considering the property gradients present in proton-irradiated material (Fig. 5). A good indicator of the presence of load partitioning is the linearity of the elastic range for the irradiated layer, which would result in an inflection upon the yielding of the non-irradiated substrate ${ }^{53}$. Although this inflection was not observed, it is difficult to state whether or not partitioning is occurring with any certainty, as there is only one measurement point between the yield stress and proportional limits of each layer. It may well be the case that the effect of partitioning is diminished due to the low volume fraction of irradiated material ( 4\%). An in-situ study of dual phase 737-DP and 775-DP steels has reported the effect of load partitioning on strain hardening was smaller in the alloy with a lower volume fraction of the harder martensite phase $\mathrm{s}^{54}$.

The strain hardening parameter of non-irradiated small-scale specimens, prepared using PFIB, has already been demonstrated to be far lower than that of bulk tests ${ }^{36}$. This is thought to be due to the high ratio of grains intersecting the surface to those fully constrained in bulk. Although the relative volumes of the irradiated specimens are approximately $50 \%$ larger than the non-irradiated, the smallest dimension is the same in both sets 
(thickness). Under the assumption that martensite block boundaries control the hardening parameter ${ }^{55,56}$, the ratio of smallest specimen thickness to "effective grain" diameter $(t / d)$ remains the same for both non-irradiated and irradiated samples. Hence, despite the larger gauge volume of the irradiated samples, they are expected to be subject to the same scale dependant diminished hardening as was observed in the non-irradiated set ${ }^{36}$. The low $\mathrm{t} / \mathrm{d}$ ratio is thought to be the origin of the diminished hardening capacity, with an increased number of grains intersecting the sample surface an easy route for dislocation annihilation is provided which moderates the rate of accumulated backstress ${ }^{57}$. Whereas, specimens with a higher $t / d$ ratio (i.e. bulk) will harden at a higher rate due to the contributions of back stresses generated by dislocations accumulating in the larger fraction of fully constrained grains ${ }^{19,58}$. It has been shown that the strain hardening parameter $(\mathrm{n})$ raises with increased $t / d$ before stabilising and accurately reflecting bulk hardening ${ }^{59}$, the position and shape of this threshold value can vary considerably between materials ${ }^{60}$.

Work by Byun and Farrell illustrates that irradiated materials exhibit a UTS that is typically similar to that of the non-irradiated material ${ }^{10,49,61-63}$. As dose is increased, the yield stress increases and is coupled with a reduction of strain hardening, at the critical point satisfying $\sigma_{\mathrm{y}} \geq \sigma_{\mathrm{u}}$ a specimen will experience prompt necking at yield. Whilst the yield shifts in the current work were insufficient to exceed this threshold value, UTS measured in specimens using both approaches were close to the one of bulk non-irradiated in all but the non-irradiated samples prepared using PFIB. Specimens prepared by top-down PFIB milling invariably exhibit side-wall tapering due to $\mathrm{Xe}^{+}$ion beam profile and material redeposition. UTS has been shown to be inversely proportional to gauge section taper in non-irradiated samples tested in tension ${ }^{36}$. This implies that taper may have been significantly lower in the micro-tensile irradiated specimens, however, they were prepared using the same methodology as in ${ }^{36}$. It may also be the case that stress has been raised above the threshold regardless of the geometric effect of taper, providing an accurate UTS.

The presented techniques are subject to intrinsic and extrinsic sources of error, some of which have already been discussed, including geometry, scale and load partitioning. Misalignment of samples in the $5 \mathrm{kN}$ loading rig used in the XRD-DIC experiments, as with all gripped sample loading rigs, can introduce scatter in recorded properties. This scatter is due to a strain gradient placing the sample in shear as a result of off-axis loading ${ }^{64}$. At the larger scale, this can be minimised by the use of guide pins, specialised grips or calibration, outlined in ASTME1012-0565. However, at the sub-millimetre length scale, specimen alignment becomes a significant issue $^{34,36,66}$. In order to ensure accurate alignment with the loading axis, specimens were prepared within the fixture that was directly attached to the tensile testing apparatus and milling was performed with care to ensure the samples remain parallel with the external fixture. Even with a well aligned gauge length, off-axis loading may result from a mismatched pair of loading contact surfaces. In a compression test this would correspond to the punch and the top of the specimen and in tension would be the loading pin and loop interior. Due to the aforementioned sidewall tapering arising from FIB milling, mismatched surfaces are inevitable without employing overtilt during preparation ${ }^{34}$.

The damage profile that is intrinsic to proton irradiated materials is also likely to introduce uncertainty in the measured properties. Each approach samples a non-linear dose which can be considered as a property gradient over the sampling range. As the softer regions near the proton-beam entry surface yield, localised deformation will occur and, even in a perfectly aligned configuration, the test will no longer be truly uniaxial. In order to avoid this localisation, the use of a significantly smaller specimen thickness than the irradiation stopping range has been suggested ${ }^{67}$, however, a smaller specimen diameter would lead to a less representative sampling of grains. This could be improved by increasing the energy of the proton beam but the advantages of an improved penetration depth must be weighed against the disadvantages of an increased activation cross section and dramatic reduction in irradiation rate. Although the sampling volume of XRD stress measurement has been shown to be weighted to the flatter region of the dose profile in this study, property gradients within the measured volume may also contribute to the error in the measurement. Non-zero out of plane shear stresses can introduce a phenomena referred to as psi-splitting, which is a breakdown in the linearity of the $\sin ^{2} \psi \operatorname{plot}^{47}$. The split manifests as an upwards and downwards deviation from the centre line at positive and negative tilts, this will increase errors in the gradient used to calculate stress. This was not observed in the present work; however, it may become apparent in specimens that possess a steeper gradient. Therefore, it may not be suitable to apply the technique to irradiation techniques yielding even a thinner irradiated layer such as heavy ion irradiation.

\section{Conclusions}

Two novel methods for testing the mechanical properties in polycrystalline samples of proton irradiated material (and potentially other surface modifications) have been applied for the first time to measure changes in yield and strain hardening behaviour and the invariance of UTS. The main conclusions are as follows:

- Both techniques have been used successfully to record stress-strain curves of proton irradiated material but significant differences in those curves were detected between the two methodologies. It is clear that additional experimentation is required to further calibrate both techniques, the most important of which are: an investigation into load partitioning between non-irradiated/irradiated layers and a systematic assessment of the smallest volume required to represent bulk behaviour.

- The combination of XRD and DIC for in-situ measurement of stress and strain provides a relatively low-cost method of recording flow curves in proton-irradiated material. The advantage to utilising this technique is that the results are comparable to those collected using standardised testing. Further sensitivity studies are necessary to identify the potential influence of the soft substrate on the yield behaviour of the irradiated layer.

- The use of $\mathrm{Xe}^{+}$PFIB has been shown to be an effective method for the manufacture of mesoscale proton irradiated specimens. However, the achievable gauge volume from a tensile sample machined in this way is still very small making it most applicable to materials with grains around the 1 micron range. In the present 

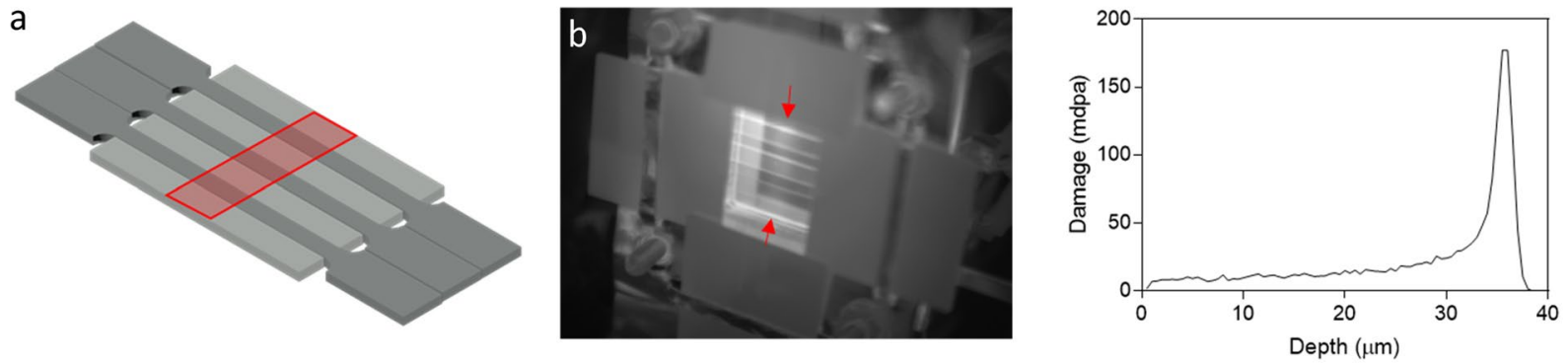

Figure 6. (a) Schematic showing the proton irradiation set up of the samples with a gauge section of $27 \times 2 \times 1$ $\mathrm{mm}^{374}$, (b) thermal image of specimens mounted on the end station during irradiation, the arrows indicate the beam position and (c) SRIM simulation for current collected on stage during proton irradiation to achieve nominal damage level of 10 mdpa.

case, SA508-4N martensitic-bainitic steel displayed a sub-micron morphology and despite such small structure the flow curves indicated a lack of constraint in the gauge volume. This is understood to be due to an insufficient number of effective dislocation barriers within the volume to facilitate the accumulation of bulk representative back stresses.

In summary, both techniques provide a method of obtaining tensile stress-strain data from the proton irradiated layer in a sampling volume that was previously impossible using preceding techniques offering complementary insights into the effect of the dose distribution on mechanical properties.

\section{Methods}

Material and specimen preparation. The material in this investigation was SA508-4N martensitic-bainitic steel, supplied by Rolls-Royce plc. Dog bone tensile samples were prepared with a $27 \mathrm{~mm} \times 2 \mathrm{~mm} \times 1 \mathrm{~mm}$ gauge section by electrical discharge machining (EDM). Coupons for the preparation of specimens by PFIB were also prepared by EDM to the dimensions $27 \mathrm{~mm} \times 3 \mathrm{~mm} \times 1 \mathrm{~mm}$. All specimens were ground to remove the recast layer and subsequently polished using a standard metallurgical preparation route.

Irradiation experiment. The irradiation experiments were carried out using the DAFNE 5MV Tandem Pelletron at the University of Manchester Dalton Cumbria Facility ${ }^{68,69}$. The specimen setup during irradiation is illustrated in Fig. 6(a,b). A "jigsaw" configuration was designed to reduce leakage of the liquid indium eutectic behind the sample used as a heat sink during irradiation. Proton irradiation was carried out at $3 \mathrm{MeV}$ at a flux of $2.3 \times 10^{14} \mathrm{~cm}^{-2} \mathrm{~s}^{-1}$ to obtain a fluence of $8.43 \times 10^{17} \mathrm{~cm}^{-2}$, with the beam rastered over a $5 \times 25 \mathrm{~mm}^{2}$ area. The beam was over-scanned by $40 \%$ onto the aperture veins to produce hard edges in the irradiated region. Proton stopping range calculations were performed using SRIM $^{70}$ with the 'quick Kinchin-Pease calculation"71. The calculation predicted a stopping peak at $\sim 36 \mu \mathrm{m}$, with the damage at $60 \%$ of the penetration depth of the stopping peak taken as the nominal damage (Fig. $6 \mathrm{c}$ ). The temperature was monitored using a pyrometer, which was pre-calibrated according to the method outlined by Wady et al. ${ }^{68}$. The mean temperature was recorded throughout the experiment as $330 \pm 3^{\circ} \mathrm{C}$. Following irradiation, specimens were lightly polished with colloidal silica in order to remove any surface implantation. The irradiated region was located by automated profile micro indentation testing, using a Struers Durascan automated indenter. A load of $0.005 \mathrm{~kg}$ was applied for 10 seconds; the use of a low load ensured indentations did not penetrate through the irradiated layer. Due to a slight aperture misalignment, the irradiated region over the sample set had a trapezoidal area. The area was measured and used to recalculate the nominal damage using the accumulated charge during irradiation. An average increase in hardness of $36 \mathrm{HV}$ was recorded in the irradiated region relative to the non-irradiated region, as shown in Fig. 7.

Method 1: XRD and DIC monitoring of plastic deformation. As the irradiated region in the gauge section was discontinuous, the specimen was further modified by EDM, as shown in Fig. 8(a). The "double dog bone" geometry was designed so that the widest point of each radius leading into the second parallel section intersected with the start and end of the irradiated region. The inner gauge section was $4 \mathrm{~mm} \times 1 \mathrm{~mm} \times 1 \mathrm{~mm}$, with a $0.5 \mathrm{~mm}$ radius in the transition region. Removal of the material ensured the experiment remained uniaxial whilst also removing the indentations used to locate the irradiated region. Samples were painted with a white anti-reflective coating and dusted over with black spray paint to apply a speckle pattern enabling the utilisation of digital image correlation (DIC) for monitoring strain.

The in-situ X-ray stress analysis and image collection strain analysis was carried out according to the methodology outlined in ${ }^{35}$. Quasistatic tensile loading was applied using a Kammrath and Weiss $5 \mathrm{kN}$ tension-compression microtester at a displacement rate of $5 \mu \mathrm{m} \mathrm{s}^{-1}$, which corresponds to a strain rate of $\sim 1.25 \times 10^{-3} \mathrm{~s}^{-1}$. The crosshead was stopped at numerous hold points throughout the test to allow for image acquisition and X-ray diffraction measurements. Optical images were analysed using the commercially available LaVision DaVis 8.1.5 image correlation software, with the total strain averaged over the second gauge section.

Single peak X-ray stress analysis was performed on a Proto portable iXRD system using the $\sin ^{2} \Psi$ technique on the $\{211\}$ reflection using $\mathrm{Cr} \mathrm{K} \alpha$ radiation giving a $2 \theta$ of $155^{\circ}$. Side inclination measurements were taken at 11 $\psi$-tilt angles between $\pm 25^{\circ}$ with 10 exposures using a $1 \mathrm{~mm}$ circular aperture. Due to the reduced aperture, the 


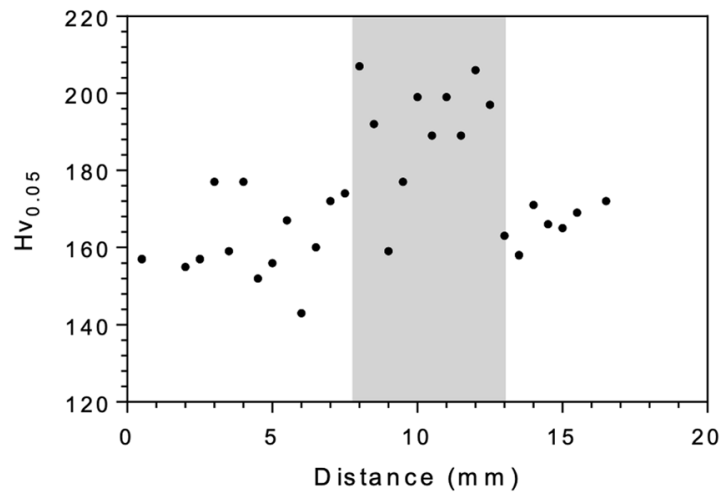

Figure 7. Typical indentation profile of irradiated region taken at $0.05 \mathrm{H}_{\mathrm{v}}(\sim 18.42 \mu \mathrm{m}$ diagonal), with an average irradiation hardening of $36 \mathrm{H}_{\mathrm{v} .}$; shaded area denotes the irradiated region.

a

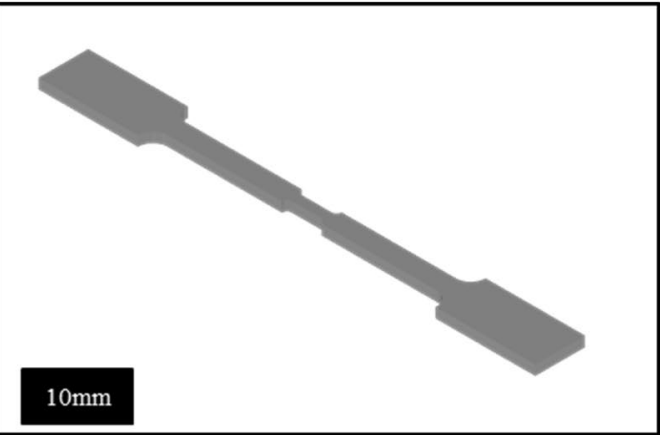

b

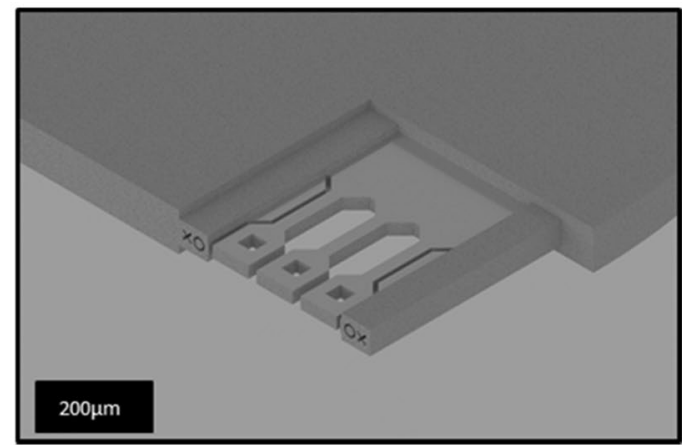

Figure 8. Specimen geometries for the two types of tensile samples (a) XRD-DIC sample, with a modified sub-gauge section of $5 \times 1 \times 1 \mathrm{~mm}^{374}$, and (b) PFIB - micro-tensile sample, with a gauge section of $\sim 150 \times 30 \times 30 \mu \mathrm{m}^{336,74}$.

counting times were increased to 3 seconds for each of the exposures and $\pm 2^{\circ}$ goniometer undulation in $\chi$ was applied to increase counting statistics. Peak position and shape was determined using a gaussian fit, with stress calculated as follows:

$$
\sigma_{x}=\left(\frac{E}{1+v}\right)_{h k l} \frac{\delta d_{x \psi}^{h k l}}{\delta \sin ^{2} \psi} \frac{1}{d_{\psi=0}}
$$

where $d_{x \psi}^{h k l}$ is the inclined lattice spacing rotated around an axis normal to the loading direction, $\mathrm{d}_{\psi=0}$ is the stress-free lattice spacing at $\psi=0^{\circ}$ and $\left(\frac{E}{1+v}\right)_{h k l}$ are the effective elastic constants of the diffracting plane (1/2 S2) calculated to be $5.94 \times 10^{-6} \mathrm{MPa}^{-135}$.

The diffraction elastic constants were obtained, using the methodology outlined in ASTM E1426 -98 ${ }^{38}$, which was to record $\operatorname{Sin}^{2} \psi$ plots for the $\{211\}$ planes at increasing/decreasing load, through a number of repeating cycles. The gradients of the $\operatorname{Sin}^{2} \psi$ are plotted against stress allowing for the calculation of the diffraction elastic constants for this grain family by the gradient of the plot. An underpinning requirement for the measurement of applied plastic stresses using $\mathrm{x}$-ray diffraction is that the interplanar response to applied stress remains linear outside of the elastic regime. A deviation in linearity during plastic deformation would cause an over or under representation of the applied stress. Selection of the correct planes for stress measurement is largely empirical but relates to the response to intergranular strains during deformation. The plane with the most linear response, with high enough intensity sitting within the measurement range of the goniometer is selected for stress measurement, conventionally it is the 211 plane family for BCC materials and the 311 family for FCC materials ${ }^{72}$.

Due to the low dose tested in this experiment, the diffraction elastic constants were assumed to be unchanged by irradiation since it is likely that point defects and dislocation loops would be present at these low doses and therefore would not significantly alter the interplanar response. In the standard case of residual stress measurement in plastically deformed surfaces using the $\operatorname{Sin}^{2} \psi$ method, such as those achieved using tooling or shot peening, the presence of dislocations does not alter the diffraction elastic constants ${ }^{73}$, so can be thought of as insensitive to this type of defect. However, in the case of materials irradiated to high doses, such as those leading to the formation of voids, precipitation and significant microstructural modification, the diffraction elastic constants would need to be calculated for this condition. 
Method 2: Plasma Focussed lon Beam (PFIB) Milling and Piezo-mechanical testing. A coupon was prepared for PFIB milling by grinding and polishing the non-irradiated face of the EDM sample. It was mounted with the irradiated face down and carefully thinned by removal of the non-irradiated side using successively finer grades of silicon carbide paper $(600,800,1200,2500$ and 4000 grit) until a thickness of $\sim 60$ $\mu \mathrm{m}$ was achieved. The foil was then carefully polished using diamond paste, starting with $3 \mu \mathrm{m}$ and finishing with $0.25 \mu \mathrm{m}$, a final polishing step was carried out using colloidal silica to ensure a mirror finish. Subsequent FIB milling is expected to have removed any surface deformation introduced by previous mechanical polishing steps. After polishing, a $3 \mathrm{~mm}$ disk was extracted from the foil using a standard transmission electron microscope specimen punch, followed by grinding with fine grit silicon carbide paper to remove burrs. The foil was mounted between two glass slides with wax and ground to apply a straight edge. Attachment of the disk to a specimen mount using cyanoacrylate adhesive readied the specimen for preparation by PFIB. Specimens were prepared using the methodology outlined in ${ }^{36}$, with all milling performed at $30 \mathrm{kV}$ and $2^{\circ}$ overtilt using a PFIB equipped FEI Helios FEG-SEM. The high current $(1.3 \mu \mathrm{A})$ ion milling was carried out from the non-irradiated side to preserve as much irradiated material as possible. This high current thinning step continued until a thickness of $\sim 40 \mu \mathrm{m}$ was reached. A subsequent automated cross polishing routine was performed using FEI Auto Slice and View 4 software package at $180 \mathrm{nA}$, removing approximately $5 \mu \mathrm{m}$ from each side. Therefore, the through thickness dimension contained only the flat portion of the dose profile with the Bragg peak removed (Fig. 6c)). A final lamella width of $400 \mu \mathrm{m}$ with a $30 \mu \mathrm{m}$ thickness provided sufficient area to prepare three parallel tensile specimens (Fig. 8b). The final specimen geometry was milled at a beam current of $180 \mathrm{nA}$ and the final gauge section dimensions were $150 \mu \mathrm{m} \times 30 \mu \mathrm{m} \times 30 \mu \mathrm{m}$, comprising of $\sim 1600$ laths given an approximate lath size of $\sim 9 \times 3 \times 3 \mu^{3}$.

Two specimens were tested to failure using a Microtesting Solutions (Hilliard, OH, USA) $\mu$-Test Rig (MTR-3), mounted in a Zeiss Sigma FEG-SEM. Loading conditions were the same as those applied in ref. ${ }^{36}$, with specimens loaded using a diamond pin at a rate of $\sim 100 \mathrm{~nm} \mathrm{~s}^{-1}$ and hold points at $200 \mathrm{~nm}$ increments. Secondary electron images were taken at each hold point to provide an accurate method of strain measurement. Strain was calculated by tracking platinum fiducial markers using digital image correlation, as outlined in ${ }^{36}$.

Received: 14 October 2019; Accepted: 2 March 2020;

Published online: 24 March 2020

\section{References}

1. Lucas, G. E. The evolution of mechanical property change in irradiated austenitic stainless steels. J. Nucl. Mater. 206, 287-305 (1993).

2. Byun, T. \& Hashimoto, N. Strain Localization In Irradiated Materials. Nucl. Eng. Technol. 38, 619-638 (2006).

3. Suzuki, K. Neutron Irradiation Embrittlement Of Asme SA508, Cl. 3 Steel. J. Nucl. Mater. 109, 443-450 (1982).

4. Was, G. S. et al. Emulation of reactor irradiation damage using ion beams. Scr. Mater. 88, 33-36 (2014).

5. Gan, J. \& Was, G. Microstructure evolution in austenitic Fe-Cr-Ni alloys irradiated with rotons: comparison with neutronirradiated microstructures. J. Nucl. Mater. 297, 161-175 (2001).

6. Li, B. S. \& Dai, Y. Tensile properties and microstructure of EC316LN irradiated in STIP-II. J. Nucl. Mater. 450, 42-47 (2014).

7. Luppo, M. I., Bailat, C., Sch, R. \& Victoria, M. Tensile properties and microstructure of $590 \mathrm{MeV}$ proton-irradiated pure Fe and a Fe \pm Cr alloy. 287, 483-487 (2000).

8. Maloy, Sa et al. Comparison of fission neutron and proton/spallation neutron irradiation effects on the tensile behavior of type 316 and 304 stainless steel. J. Nucl. Mater. 318, 283-291 (2003).

9. Maloy, Sa et al. The high temperature three point bend testing of proton irradiated 316L stainless steel and $\mathrm{Mod} 9 \mathrm{Cr}-1 \mathrm{Mo}$. J. Nucl. Mater. 343, 191-196 (2005).

10. Byun, T. S. Dose dependence of true stress parameters in irradiated bcc, fcc, and hcp metals. J. Nucl. Mater. 361, 239-247 (2007).

11. Tong, Z. \& Dai, Y. Tensile properties of the ferritic martensitic steel F82H after irradiation in a spallation target. J. Nucl. Mater. 385 , 258-261 (2009).

12. Saito, S. et al. Proton irradiation effects on tensile and bend-fatigue properties of welded F82H specimens. J. Nucl. Mater. 398, 49-58 (2010).

13. Sencer, B. H. et al. Proton irradiation emulation of PWR neutron damage microstructures in solution annealed 304 and cold-worked 316 stainless steels. J. Nucl. Mater. 323, 18-28 (2003).

14. Gupta, G., Jiao, Z., Ham, aN., Busby, J. T. \& Was, G. S. Microstructural evolution of proton irradiated T91. J. Nucl. Mater. 351, 162-173 (2006)

15. Was, G. S. et al. Emulation of neutron irradiation effects with protons: validation of principle. J. Nucl. Mater. 300, 198-216 (2002).

16. Rafique, M., Chae, S. \& Kim, Y.-S. Surface, structural and tensile properties of proton beam irradiated zirconium. Nucl. Instruments Methods Phys. Res. Sect. B Beam Interact. with Mater. Atoms 368, 120-128 (2016).

17. Ahmad, S. et al. Effect of ion irradiation on the surface, structural and mechanical properties of brass. Nucl. Instruments Methods Phys. Res. Sect. B Beam Interact. with Mater. Atoms 325, 5-10 (2014).

18. Kiener, D., Hosemann, P., Maloy, S. A. \& Minor, A. M. In situ nanocompression testing of irradiated copper. Nat. Mater. 10, 608-13 (2011).

19. Jin, H.-H., Ko, E., Kwon, J., Hwang, S. S. \& Shin, C. Evaluation of critical resolved shear strength and deformation mode in protonirradiated austenitic stainless steel using micro-compression tests. J. Nucl. Mater. 470, 155-163 (2016).

20. Grieveson, E. M., Armstrong, D. E. J., Xu, S. \& Roberts, S. G. Compression of self-ion implanted iron micropillars. J. Nucl. Mater. 430, 119-124 (2012).

21. Sharon, Ja, Hattar, K., Boyce, B. L. \& Brewer, L. N. Compressive Properties of $<110>$ Cu Micro-Pillars after High-Dose Self-Ion Irradiation. Mater. Res. Lett. 2, 57-62 (2014).

22. Reichardt, A. et al. In situ micro tensile testing of $\mathrm{He}+2$ ion irradiated and implanted single crystal nickel film. Acta Mater. 100, 147-154 (2015).

23. Kiener, D., Grosinger, W., Dehm, G. \& Pippan, R. A further step towards an understanding of size-dependent crystal plasticity: In situ tension experiments of miniaturized single-crystal copper samples. Acta Mater. 56, 580-592 (2008).

24. Odette, G. R., He, M., Gragg, D., Klingensmith, D. \& Lucas, G. E. Some recent innovations in small specimen testing. J. Nucl. Mater. 307-311, 1643-1648 (2002).

25. Busby, J. T., Hash, M. C. \& Was, G. S. The relationship between hardness and yield stress in irradiated austenitic and ferritic steels. J. Nucl. Mater. 336, 267-278 (2005).

26. Katoh, Y., Ando, M. \& Kohyama, a Radiation and helium effects on microstructures, nano-indentation properties and deformation behavior in ferrous alloys. J. Nucl. Mater. 323, 251-262 (2003).

27. Hosemann, P. et al. Nanoindentation on ion irradiated steels. J. Nucl. Mater. 389, 239-247 (2009). 
28. Liu, X. et al. Evaluation of radiation hardening in ion-irradiated Fe based alloys by nanoindentation. J. Nucl. Mater. 444, 1-6 (2014).

29. Uchic, M. D., Dimiduk, D. M., Florando, J. N. \& Nix, W. D. Sample dimensions influence strength and crystal plasticity. Science (80.). 305, 986-989 (2004).

30. Akarapu, S., Zbib, H. M. \& Bahr, D. F. Analysis of heterogeneous deformation and dislocation dynamics in single crystal micropillars under compression. Int. J. Plast. 26, 239-257 (2010).

31. Kiener, D., Motz, C. \& Dehm, G. Micro-compression testing: A critical discussion of experimental constraints. Mater. Sci. Eng. A 505, 79-87 (2009).

32. Frick, C. P., Clark, B. G., Orso, S., Schneider, A. S. \& Arzt, E. Size effect on strength and strain hardening of small-scale $\left[\begin{array}{llll}1 & 1 & 1\end{array}\right]$ nickel compression pillars. Mater. Sci. Eng. A 489, 319-329 (2008).

33. C.K.MIYAHARA, TADA., C., UDA, T. \& Igata, N. The effects of grain and specimen sizes on mechanical properties of type 316 austenitic stainless steel. J. Nucl. Mater. 30, 1-5 (1985).

34. Wheeler, R., Pandey, A., Shyam, A., Tan, T. \& Lara-Curzio, E. Small Scale Mechanical Characterization of Thin Foil Materials via Pin Load Microtesting. Exp. Mech. 55, 1375-1387 (2015).

35. Smith, A. D. et al. Applying a combination of Laboratory X-Ray Diffraction and Digital Image Correlation for Recording Uniaxial Stress-Strain Curves in Thin Surface Layers. Submitt. to Int. J. Mech. Sci. (2019).

36. Smith, A. D. et al. On the application of Xe+ plasma FIB for micro-fabrication of small-scale tensile specimens. Exp. Mech. (2019).

37. Foecke, T., Iadicola, Ma, Lin, a \& Banovic, S. W. A Method for Direct Measurement of Multiaxial Stress-Strain Curves in Sheet Metal. Metall. Mater. Trans. 38, 306-313 (2007).

38. American Society for Testing Materials. Standard Method for Determining the Effective Elastic Parameter for X-Ray Diffraction Measurements of Residual Stress. ASTM Int. (2003).

39. Burnett, T. L. et al. Large volume serial section tomography by Xe Plasma FIB dual beam microscopy. Ultramicroscopy 161, 119-129 (2016).

40. Menon, R. \& Nabhiraj, P. Y. High speed micro-fabrication using inductively coupled plasma ion source based focused ion beam system. Vacuum 111, 166-169 (2015).

41. Garnier, A., Filoni, G., Hrnčír, T., Hladík, L. \& Plasma, F. I. B. Enlarge your field of view and your field of applications. Microelectron. Reliab. 55, 2135-2141 (2015).

42. Tucker, R. P. Dislocation Channeling in Neutron-Irradiated Niobium. J. Appl. Phys. 40, 400 (1969).

43. Kelley, R. D., Song, K., Van Leer, B., Wall, D. \& Kwakman, L. Xe+ FIB Milling and Measurement of Amorphous Silicon Damage. Microsc. Microanal. 19, 862-863 (2013).

44. Giannuzzi, L. \& Smith, N. TEM Specimen Preparation with Plasma FIB Xe+ Ions. Microsc. Microanal. 17, 646-647 (2011).

45. Ishitani, T. \& Kaga, H. Calculation of Local Temperature Rise in Focused-Ion-Beam Sample Preparation. J. Electron Microsc. (Tokyo). 44, 331-336 (1995).

46. Nikolaeva, A. V. Restoration of Mechanical Properties of Irradiated Steel by Thermal Annealing. At. Energy 90, 457-460 (2001)

47. Noyan, I. \& Cohen, J. Residual stress: Measurement by Diffraction and Interpretation. (1987).

48. Ohr, S. M. Work hardening characteristics of neutron irradiated iron. Scr. Metall. 2, 213-216 (1968).

49. Sang Byun, T., Farrell, K. \& Li, M. Deformation in metals after low-temperature irradiation: Part II - Irradiation hardening, strain hardening, and stress ratios. Acta Mater. 56, 1056-1064 (2007).

50. Withers, P. J. \& Clarke, A. P. A neutron diffraction study of load partitioning in continuous Ti/SiC composites. Acta Mater. 46, 6585-6598 (1998).

51. Neov, D. et al. In situ Neutron Diffraction Study of $\alpha-\gamma \mathrm{Fe}-\mathrm{Cr}-$ Ni. Acta Mater. 49, 2471-2479 (2001).

52. Woo, W. et al. Stress-strain relationship between ferrite and martensite in a dual-phase steel studied by in situ neutron diffraction and crystal plasticity theories. Acta Mater. 60, 6972-6981 (2012).

53. Daymond, M. R., Hartig, C. \& Mecking, H. Interphase and intergranular stress generation in composites exhibiting plasticity in both phases. Acta Mater. 53, 2805-2813 (2005).

54. Morooka, S., Sato, N., Ojima, M. \& Harjo, S. In-situ Neutron Diffraction Study on Work-hardening Behavior in a Ferrite-Martensite Dual Phase Steel. Int. J. Automot. Eng. 2, 131-136 (2011).

55. Shibata, A., Nagoshi, T., Sone, M., Morito, S. \& Higo, Y. Evaluation of the block boundary and sub-block boundary strengths of ferrous lath martensite using a micro-bending test. Mater. Sci. Eng. A 527, 7538-7544 (2010).

56. Ohmura, T., Minor, A. M., Stach, E. A. \& Morris, J. W. Dislocation-grain boundary interactions in martensitic steel observed through in situ nanoindentation in a transmission electron microscope. J. Mater. Res. 19, 3626-3632 (2004).

57. Keller, C. \& Hug, E. Kocks-Mecking analysis of the size effects on the mechanical behavior of nickel polycrystals. Int. J. Plast. 98, 106-122 (2017)

58. Keller, C., Hug, E., Retoux, R. \& Feaugas, X. TEM study of dislocation patterns in near-surface and core regions of deformed nickel polycrystals with few grains across the cross section. Mech. Mater. 42, 44-54 (2010).

59. Igata, N., Kohno, Y. \& Fujihira, T. Mechanical Properties of Thin-Foil Specimens. In Strength of Metals and Alloys. 1, 39-43 (1985).

60. Kumar, K. et al. Optimisation of thickness of miniature tensile specimens for evaluation of mechanical properties. Mater. Sci. Eng. A 675, 32-43 (2016).

61. Byun, T. S., Farrell, K. \& Li, M. Deformation in metals after low-temperature irradiation: Part I - Mapping macroscopic deformation modes on true stress-dose plane. Acta Mater. 56, 1044-1055 (2007).

62. Byun, T. S. \& Farrell, K. Plastic instability in polycrystalline metals after low temperature irradiation. Acta Mater. 52, 1597-1608 (2004).

63. Byun, T. S. \& Farrell, K. Irradiation hardening behavior of polycrystalline metals after low temperature irradiation. J. Nucl. Mater. 326, 86-96 (2004).

64. J.R Davis. Tensile testing. (ASM International (1992).

65. ASTM. Standard practice for verification of testing frame and specimen alignment under tensile and compressive axial force application. E1012 - 14 i, 1-18 (2013).

66. Zhang, H., Schuster, B. E., Wei, Q. \& Ramesh, K. T. The design of accurate micro-compression experiments. Scr. Mater. 54, 181-186 (2006).

67. Kiener, D. et al. Application of small-scale testing for investigation of ion-beam-irradiated materials. J. Mater. Res. 27, 2724-2736 (2012).

68. Wady, P. T. et al. Accelerated radiation damage test facility using a $5 \mathrm{MV}$ tandem ion accelerator. Nucl. Instruments Methods Phys. Res. Sect. A. Accel. Spectrometers, Detect. Assoc. Equip. 806, 109-116 (2015).

69. Leay, L. et al. Development of irradiation capabilities to address the challenges of the nuclear industry. Nucl. Instruments Methods Phys. Res. Sect. B. Beam Interact. with Mater. Atoms 343, 62-69 (2015).

70. Ziegler, J. F., Ziegler, M. D. \& Biersack, J. P. SRIM - The stopping and range of ions in matter (2010). Nucl. Instruments Methods Phys. Res. Sect. B. Beam Interact. with Mater. Atoms 268, 1818-1823 (2010).

71. Stoller, R. E. et al. On the use of SRIM for computing radiation damage exposure. Nucl. Instruments Methods Phys. Res. Sect. B. Beam Interact. with Mater. Atoms 310, 75-80 (2013).

72. Clausen, B., Leffers, T. \& Lorentzen, T. On the proper selection of reflections for the measurement of bulk residual stresses by diffraction methods. Acta Mater. 51, 6181-6188 (2003).

73. James, M. R. \& Cohen, J. The Measurement of Residual Stresses by X-Ray Diffraction Techniques. Exp. Methods Mater. Sci. I (1978).

74. Autodesk. AutoCAD, https://www.autodesk.com/ (2017). 


\section{Acknowledgements}

The authors would like to thank the EPSRC for funding through EP/J021172/1. This work was supported by the Henry Royce Institute for Advanced Materials, funded through EPSRC grants EP/R00661X/1, EP/S019367/1, EP/ P025021/1 and EP/P025498/1. Philip J Withers is grateful for funding through European Research Council Grant CORREL-CT (No. 695638). Allan Harte would like to acknowledge EPSRC Grant EP/T012250/1 for time and resources. The authors would also like to thank Felicity Baxter, Sandeep Irukuvarghula, Imran Bhamji, Samir de Moraes Shubeuta, Paul Wady, Joseph Henry Ward, Rhys Thomas and Rebecca Jones for their valuable assistance during the irradiation experiment.

\section{Author contributions}

Albert Smith performed XRD \& DIC testing, coordinated work, wrote main body of manuscript and analysis of all results. Jack M. Donoghue operated the PFIB, facilitated tensile testing of PFIB prepared samples and carried out orientation mapping. Alistair J.W. Garner operated the PFIB. David Lunt and Allan Harte contributed significantly to the irradiation experiments. Keith Wilford, Philip J. Withers and Michael Preuss supervised the lead author.

\section{Competing interests}

The authors declare no competing interests.

\section{Additional information}

Correspondence and requests for materials should be addressed to A.D.S.

Reprints and permissions information is available at www.nature.com/reprints.

Publisher's note Springer Nature remains neutral with regard to jurisdictional claims in published maps and institutional affiliations.

(c) (i) Open Access This article is licensed under a Creative Commons Attribution 4.0 International License, which permits use, sharing, adaptation, distribution and reproduction in any medium or format, as long as you give appropriate credit to the original author(s) and the source, provide a link to the Creative Commons license, and indicate if changes were made. The images or other third party material in this article are included in the article's Creative Commons license, unless indicated otherwise in a credit line to the material. If material is not included in the article's Creative Commons license and your intended use is not permitted by statutory regulation or exceeds the permitted use, you will need to obtain permission directly from the copyright holder. To view a copy of this license, visit http://creativecommons.org/licenses/by/4.0/.

(C) The Author(s) 2020 